\title{
As Temáticas em Saúde na Prática Pedagógica de Professores de Educação Física de um Município do Estado de Alagoas (Brasil)
}

\author{
Health Themes in Pedagogical Practice of Physical Education Teachers from a city in Alagoas \\ State (Brazil)
}

\section{Los Temas en Salud en la Práctica Pedagógica de los Maestros de Educación Física de un Municipio del Estado de Alagoas (Brasil)}

\author{
Madson Marcondes Rocha ${ }^{\mathrm{I}}$, Antonio Filipe Pereira Caetano ${ }^{\mathrm{II}}$
}

\begin{abstract}
Resumo
O ensino dos temas em saúde é uma das principais responsabilidades do professor de educação física na educação básica. As orientações para essa prática pedagógica já foram apontadas em documentos específicos, como nos Parâmetros Curriculares Nacionais (PCN'S) e na Base Nacional Comum Curricular (BNCC). O objetivo do artigo é identificar se os temas em saúde têm aparecido na prática pedagógica dos professores de educação física escolar da Educação Básica no Munícipio de Marechal Deodoro - AL. A amostra é composta por 20 docentes da rede pública municipal. Os dados foram coletados através de questionário semiestruturado online para os temas em saúde apresentados. Os resultados foram analisados a partir da estatística descritiva, expressos em média e desvio padrão. Observou-se que as dimensões de Atividade Física, Corpo e Saúde e Alimentação aparentaram escores mais positivos. Enquanto isso, os itens Comportamento de Risco, Controle do Estresse e Sociedade e Natureza apontaram para uma frequência regular. Já a dimensão Testes e Medidas foi aquela que apresentou os dados mais negativos.
\end{abstract}

Palavras chave: Ensino de Educação Física; Saúde na Escola; Currículo Escolar

\begin{abstract}
The teaching of health topics is one of the main responsibilities of the Physical Education teacher in Basic Education. The guidelines for this pedagogical practice have already been pointed out in specific documents such as the National Curriculum Parameters (PCN'S) and the National Common Curricular Base (BNCC). The aim of the article is to identify whether health issues have appeared in pedagogical practice of Physical Education teachers in Basic Education in Marechal Deodoro city, Alagoas State (Brazil). The sample consisted of twenty teachers from a public school system. Data were collected through an online semi-structured questionnaire for the health topics presented. The results were analyzed using descriptive statistics, expressed as mean and standard deviation. It was observed that the dimensions of Physical Activity, Body and Health and Food appeared to have more positive scores. Meanwhile, the items Risky Behavior, Stress Control and Society and Nature pointed to a regular frequency. The dimension Tests and Measures was the one that presented the most negative data.
\end{abstract}

Keywords: Physical Education Teaching; Health at School; School Curriculum

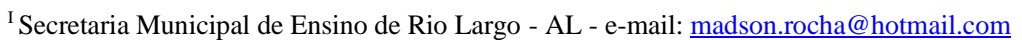

${ }^{\text {II }}$ Universidade Federal de Alagoas - UFAL - Endereço: Av. Lourival Melo Mota, S/N Tabuleiro do Martins Maceió - AL, CEP 57072-900, Brasil - e-mail: filipe.caetano@iefe.ufal.br
} 


\section{Resumen}

La enseñanza de temas de salud es una de las principales responsabilidades del docente de educación física en la Educación Básica. Los lineamientos para esta práctica pedagógica ya han sido señalados en documentos oficiales de Brasil como en los Parámetros Curriculares Nacionales (PCN'S) y en la Base Curricular Común Nacional (BNCC). El objetivo del artículo es identificar si los temas en salud han aparecido en la práctica pedagógica de los docentes de educación física de Educación Básica del municipio de Marechal Deodoro estado de Alagoas, Brasil. La muestra ha sido formada por 20 docentes del sistema de escuelas públicas municipales. Los datos fueron recolectados a través de un cuestionario semiestructurado en línea para los temas de salud presentados. Los resultados se analizaron mediante estadística descriptiva, expresada por la media y desviación estándar. Se observó que las dimensiones de Actividad Física, Cuerpo y Salud y Alimentación parecían tener puntuaciones más positivas. Mientras tanto, los ítems Comportamiento de Riesgo, Control del Estrés y Sociedad y Naturaleza apuntaban a una frecuencia regular. La dimensión Pruebas y Medidas fue la que presentó los datos más negativos.

Palabras clave: Enseñanza de Educación Física; Salud en la escuela; Currículo Escolar

\section{Introdução}

A saúde foi estudada como elemento unidimensional e caracterizada pela ausência de doença. A partir do século XX, após vários debates, a Organização Mundial da Saúde (OMS) definiu saúde como um estado de completo bem-estar físico, psicológico e social. O conceito recebeu inúmeras críticas por tratar a saúde como um conceito pessoal e abstrato, entendido como utópico e inalcançável, mas enfatizava a saúde como uma responsabilidade social de diversos setores governamentais (OMS, 2006).

A saúde é garantida por leis nas diferentes esferas sociais e nos divergentes ciclos da vida. A Constituição Federal (BRASIL,1988) citou-a como um direito de todos e um dever do Estado, sendo o principal marco para criação do Sistema Único de Saúde (SUS). O Estatuto da Criança e do Adolescente (ECA) trouxe o enfoque na proteção integral, sem distinção de raça, cor ou classe social (BRASIL, 2010).

Dentre os documentos disponíveis pelo SUS para promoção da saúde de crianças e adolescentes, destacam-se a Política Nacional de Atenção Integral à Saúde da Criança (PNAISC) e as Diretrizes Nacionais para a Atenção Integral à Saúde de Adolescentes e Jovens na Promoção, Proteção e Recuperação da Saúde (PNAISAJ). O PNAISC enfoca desde a atenção humanizada até a vigilância em saúde no âmbito físico, social e comportamental de 0 a 9 anos (BRASIL, 2018). O PNAISAJ, com recomendações para indivíduos entre 10 e 24 anos, volta-se para a promoção da saúde baseada nas condições de gênero, direitos sexuais, ética, cidadania, igualdade racial e cultura da paz (BRASIL, 2010)

Considerando a saúde da criança e do adolescente como prioridade, a escola recebeu atenção especial, por ser, depois da residência, o segundo local onde esses indivíduos passam a maior parte do tempo. Em 2007, o Governo Federal lançou o Programa Saúde na Escola (PSE) com o objetivo de 
contribuir para a formação integral dos escolares por meio de ações específicas de intervenção e de capacitação dos profissionais. Dentre os programas executados pelo PSE destacou-se "Crescer Saudável", uma ação que visou à prevenção, ao controle e ao tratamento da obesidade infantil (BRASIL, 2010).

Segundo a Lei de Diretrizes e Bases da Educação (LDB) "a educação tem por finalidade o pleno desenvolvimento do educando, seu preparo para o exercício da cidadania e sua qualificação para o trabalho". É impossível cumprir essa finalidade sem uma educação em saúde. Desta forma, os documentos norteadores curriculares para os diferentes níveis de ensino citam a saúde como ponto fundamental (BNCC, 2019). A LDB apresentou uma discussão sobre os direitos e deveres dos escolares, mas as únicas menções pontuais foram a obrigatoriedade da educação física nas instituições de ensino, a inclusão dos conteúdos "direitos humanos e à prevenção de todas as formas de violência" e "educação alimentar e nutricional" nos temas transversais de ensino (BRASIL, 2018).

Os Parâmetros Curriculares Nacionais (PCN's) destacaram a importância da saúde na escola e apresentaram a relevância em explorar esse conteúdo em documentos específicos (BRASIL, 1998). Estes fornecem embasamento para os profissionais da educação construírem seus planejamentos didáticos a respeito do tema, apresentando conteúdos, objetivos e critérios de avaliação para o início do Ensino Fundamental.

Atualmente, a Base Nacional Comum Curricular (BNCC) com uma perspectiva de Competências e Habilidades, citou a temática da saúde diversas vezes. Na primeira parte do texto, em que apresenta as competências e as habilidades gerais para a educação, a competência $\mathrm{N}^{0} 8$ apontava: "conhecer-se, apreciar-se e cuidar de sua saúde física e emocional, compreendendo-se na diversidade humana e reconhecendo suas emoções e as dos outros, com autocrítica e capacidade para lidar com elas" (BRASIL, 2018).

No entanto, mesmo com diversas diretrizes para promoção da saúde, os dados da última Pesquisa Nacional de Saúde do Escolar (PeNSE) apontaram para resultados nada animadores para os indicadores em saúde dos escolares, sobretudo revelando a prevalência do sedentarismo, bem como outros comportamentos de risco que interferem na saúde do indivíduo (IBGE, 2015). Dito isso, surgiu a problemática: com que frequência os professores de Educação Física têm abordado as temáticas sobre saúde durante suas aulas nas escolas? Assim, o objetivo do presente estudo foi identificar se os temas em saúde têm aparecido na prática pedagógica dos professores de educação física escolar da Educação Básica no Munícipio de Marechal Deodoro do Estado de Alagoas. 


\section{Metodologia}

\subsection{Tipo de Pesquisa}

O presente estudo configura-se como quantitativo, descritivo, de caráter exploratório, de corte transversal (MINAYO; SANCHES, 1993).

Esta pesquisa faz parte do projeto “(Re) PeNSE Alagoas: Saúde e Educação Física Escolar na Educação Básica”, em andamento e aprovado pelo Comitê de Ética da Universidade Federal de Alagoas (CEP-UFAL) sob o $\mathrm{N}^{\mathrm{o}}$ de Protocolo 3.777.174/2019.

\subsection{População e Amostra}

A prefeitura do Município de Marechal Deodoro/ AL possui 516 professores (contratados e efetivos), atuando em 17 escolas que atendem a 9.317 alunos em todo Ensino Fundamental. Desse corpo docente, 28 são professores da disciplina curricular obrigatória de educação física e, desses, apenas 23 (14 contratados e 9 efetivos), estão efetivamente atuando em sala de aula.

A amostra foi escolhida por conveniência. Foram incluídos os professores graduados, concursados ou contratados, atuando em escola pública do município em 2019, de ambos os sexos, em quaisquer níveis da Educação Básica, independentemente do tempo de formação e atuação profissional.

Foram excluídos da amostra os professores que tenham deixado alguma questão do questionário em branco, que não assinaram o Termo de Consentimento Livre e Esclarecido (TCLE) e/ ou desistiram de fazer parte da pesquisa ao longo do estudo.

\subsection{Procedimentos de Coleta}

No primeiro momento, foi feito o contato com a Secretaria Municipal de Educação (SEMED-MD) para assentimento da realização da pesquisa e coleta dos dados dos professores. Em seguida, realizado o contato com os docentes, convidando-os a participarem da pesquisa, e o agendamento do preenchimento do questionário. Por fim, o instrumento (construído na plataforma google forms) foi compartilhado presencialmente com o participante do estudo, momento em que também foi recolhida a assinatura no 
TCLE. Enquanto os participantes preenchiam o formulário, o pesquisador ficou à disposição para sanar possíveis dúvidas.

Depois do preenchimento do questionário foram tabulados os dados no programa Excel 16.0 para a realização da análise dos dados.

\subsection{Instrumento de Pesquisa}

As variáveis de interesse desse estudo foram os temas sobre saúde na prática pedagógica dos professores de educação física. Tais conteúdos foram identificados a partir das diretrizes do Programa de Saúde na Escola (PSE); da Pesquisa Nacional de Saúde do Escolar (PeNSE); do Global School-Based Student Health Survey (GSHS); da BNCC; dos PCN's; do guideline Instrumental Activities of Daily Living (IADLs) e nos Determinantes Sociais de Saúde (DSS) da Saúde Coletiva do Ministérios da Saúde.

Utilizou-se um questionário misto (aberto/ fechado e com escalas), com 24 itens, apresentado na plataforma Google Docs, com questões divididas em cinco partes: dados pessoais (5 Itens), dados profissionais ( 5 itens), experiência no ensino de saúde (5 itens), temas em saúde ( 7 dimensões) e prática pedagógica profissional (2 itens).

No componente Temas em Saúde as dimensões escolhidas foram: alimentação, atividade física e comportamento sedentário; comportamentos de risco à saúde; controle do estresse e relações interpessoais; sociedade e natureza; corpo e saúde. No componente Prática Pedagógica Profissional foram identificados os conteúdos da educação física escolar mais aplicados e a realização de testes e medidas voltados para a saúde do aluno.

Os itens foram respondidos em escala tipo Likert, que buscava mensurar atitudes através de opiniões objetivas sobre determinado tema (LUCIAN, 2016). O participante poderia responder nunca, raramente, quase sempre ou sempre, tendo como referência a realização de atividades teóricas e/ ou práticas nos últimos seis meses.

\subsection{Análise dos Dados}

Os subitens das dimensões dos Temas em Saúde foram computados através de valores 0 e 1 associados ao perfil negativo para as temáticas de saúde, correspondendo às respostas nunca e raramente. 
Os valores 2 e 3 foram associados ao perfil positivo, correspondendo as respostas quase sempre e sempre. Não houve a inclusão da opção sem opinião. A definição das bases dos escores teve como referências as informações dispostas por Dalmoro \& Vieira (2013).

Os escores para as dimensões foram descritos em médias a partir da soma das pontuações atribuídas às respostas, divididas pela quantidade de perguntas daquela dimensão. Os resultados entre 0 e 0,99 foram consideradas ações negativas; entre 1 e 1,99 ações regulares; e entre 2 e 3 ações positivas para temáticas em saúde na prática docente.

Os dados foram analisados através de estatística descritiva (REIS, 2002), com apresentação de média e desvio padrão, apresentados em tabelas de distribuição de frequência e gráficos.

\section{Resultados e Discussão}

\subsection{Perfil de Formação e Social dos Professores}

Foram entrevistados 20 professores de educação física da rede municipal de ensino de Marechal Deodoro - AL (SEMED-MD), correspondendo a 71,4\% dos professores de educação física da rede municipal, conforme demonstra a figura 1.

Figura 1 - Amostra dos Professores de Educação Física da SEMED-MD/2019

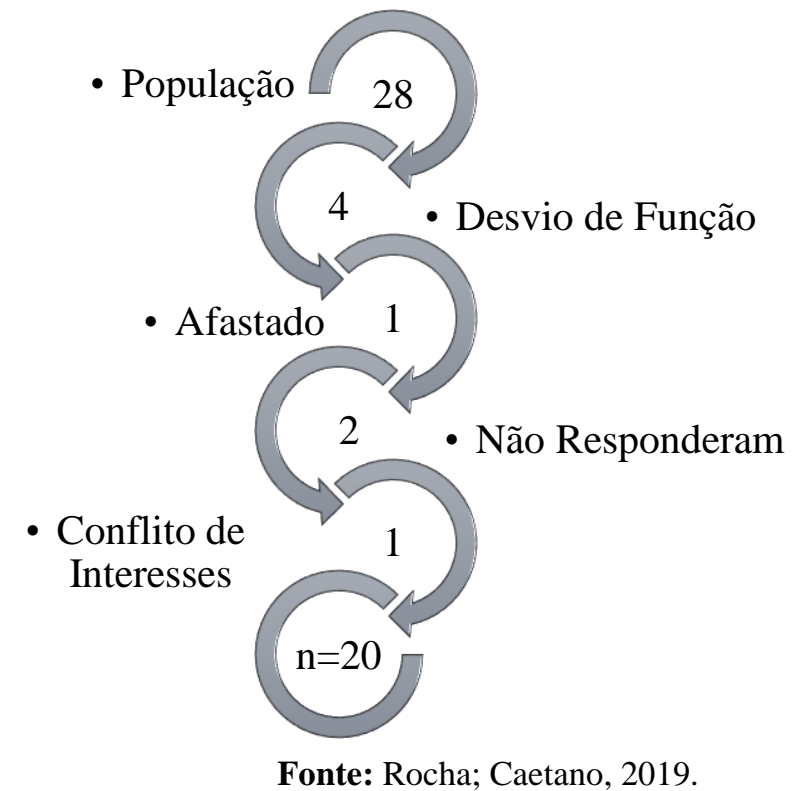

Revista Kinesis, Santa Maria, v. 39, p.01-19, 2021 
A coleta foi realizada entre 4 e 30 de novembro de 2019, momento em que nem todos os docentes estavam em atividades em sala de aula. A amostra foi composta por professores entre 24 e 57 anos, graduados em instituições de ensino superior públicas e privadas, com variação de tempo de atuação de $1 \pm 33$ anos, e que atuavam em vários níveis da Educação Básica (tabela 1).

Tabela 1 - Perfil social e profissional dos Professores de Educação Física da SEMED-MD.

\begin{tabular}{|c|c|c|}
\hline Variável & $\mathbf{N}$ & $\%$ \\
\hline \multicolumn{3}{|l|}{ Sexo } \\
\hline Masculino & 10 & $50 \%$ \\
\hline Feminino & 10 & $50 \%$ \\
\hline \multicolumn{3}{|l|}{ Vínculo Empregatício } \\
\hline Concurso Público & 8 & $40 \%$ \\
\hline Contrato Temporário & 12 & $60 \%$ \\
\hline \multicolumn{3}{|l|}{ Quantidade de Escolas de Atuação } \\
\hline 1 & 12 & $60 \%$ \\
\hline 2 & 6 & $30 \%$ \\
\hline 3 & 2 & $10 \%$ \\
\hline \multicolumn{3}{|l|}{ Instituição de Graduação } \\
\hline Pública & 8 & $40 \%$ \\
\hline Privada & 12 & $60 \%$ \\
\hline \multicolumn{3}{|l|}{ Nível de Formação } \\
\hline Graduação & 7 & $35 \%$ \\
\hline Especialização & 12 & $60 \%$ \\
\hline Mestrado & 1 & $5 \%$ \\
\hline \multicolumn{3}{|l|}{ Níveis de Atuação } \\
\hline Fundamental Anos Iniciais & 14 & $70 \%$ \\
\hline Fundamental Anos Finais & 17 & $85 \%$ \\
\hline Educação de Jovens e Adultos & 5 & $25 \%$ \\
\hline \multicolumn{3}{|l|}{ Tempo de Atuação Profissional } \\
\hline Até 1 ano & 1 & $5 \%$ \\
\hline $1-3$ Anos & 3 & $15 \%$ \\
\hline 5-7 Anos & 3 & $15 \%$ \\
\hline $7-9$ Anos & 4 & $20 \%$ \\
\hline+10 Anos & 9 & $45 \%$ \\
\hline
\end{tabular}

Fonte: Rocha; Caetano, 2019.

No que tange a existência de disciplinas relacionadas à saúde dentro do currículo de formação profissional em educação física dos participantes, 90\% deles (18 professores) relataram ter informações sobre promoção da saúde, qualidade de vida e bem-estar, em contraposição a 10\% (2) que informaram não ter tido qualquer tipo de disciplina com este conteúdo em sua formação.

A prática do professor de educação física, sobretudo após a LDB, provocou reflexões acerca da formação desses profissionais no Ensino Superior. O currículo sofreu modificações ao longo dos anos, 
contribuindo para a formação das chamadas "crises" de identidade na área (COLETIVO DE AUTORES, 1992). Apesar das mudanças, o tema saúde sempre esteve intrínseco à formação, e, assim, as disciplinas sobre a temática sempre estiveram presentes. Atualmente, uma das mudanças que mais influenciaram na construção dos currículos dos cursos de formação em educação física foi a separação das áreas de atuação, considerando o bacharel como profissional que atua em academias, clubes e serviços da atenção básica à Saúde e o licenciado como professor na Escola.

Essa mudança retirou das matrizes curriculares dos cursos de licenciatura muitas disciplinas relacionadas à área da saúde, substituindo-as por disciplinas de conteúdo pedagógico e/ ou das áreas humanas. Essa ausência pode ter provocado uma formação deficitária de profissionais que acabavam recorrendo a outras práticas para aquisição deste conhecimento. Para Oliveira et al., "verifica-se a necessidade da presença de um professor de educação física capaz de articular e mediar questões atuais de modo a proporcionar elementos na construção de fundamentos para a saúde” (2014, p.10). Quando os professores não recebem uma formação adequada sobre o tema saúde, acabam reféns de projetos específicos das instituições e de iniciativas pontuais, como afirmam Lima, Malacarne, Strieder:

O desconhecimento dos professores em geral, sobre as questões mais básicas se enraíza no processo de formação inicial e se alonga na formação continuada. No interior da escola, a ausência de projetos amplos direcionados pelos professores, cuja formação é insuficiente para desenvolver o tema, a educação em saúde é por vezes amenizada por visitas técnicas de profissionais de saúde [...] $(2012$, p.12)

Mesmo os professores que informaram não ter esses conteúdos em sua formação, apontaram se sentirem capacitados para desenvolverem atividades sobre a temática da saúde durante suas aulas de educação física. Como justificativa, destacaram o conteúdo saúde como uma temática intrínseca à área, bem como as possibilidades de ampliação da formação a partir de cursos, seminários, estudos individuais e palestras.

\subsection{Temas em Saúde nas Aulas de Educação Física}

A presença dos temas em saúde nas aulas dos professores de educação física do município de Marechal Deodoro/AL não se manifestou de forma equânime. As dimensões de Atividade Física, Corpo e Saúde e Alimentação aparentaram escores mais positivos. Enquanto isso, os itens Comportamento de Risco, Controle do Estresse e Sociedade e Natureza apontaram para uma frequência regular. Já a dimensão Testes e Medidas foi aquela que apresentou os dados mais negativos. 
Os estudos sobre a frequência das temáticas em saúde nas aulas de educação física não existem. A busca nas bases de dados (Scielo, Scopus e Eric) para identificar a existência destas investigações demonstrou a lacuna desta temática no diálogo com a produção científica. No âmbito da prática pedagógica escolar e da temática saúde, as pesquisas versam sobre o conhecimento docente dos conceitos de saúde, promoção da saúde, bem-estar e qualidade de vida (BANKOFF \& ZAMAI, 1999 ; GUIMARÃES, NEIRA \&VELARDI, 2015); dos fatores de risco à saúde (PAIXÃO, AGUIAR \& SILVEIRA, 2015; ROMBALDI et. al., 2012); e das abordagens pedagógicas como saúde renovada e promoção da saúde (KNUTH, AZEVEDO \& RIGO, 2006; ZACHA et. Ali, 2013; JESUS \& SAWITZKI, 2015, TEIXEIRA \& COLOMBO, 2020). Logo, são estudos mais voltados à busca de aspectos conceituais do que à procura de perspectivas procedimentais e atitudinais na prática pedagógica docente no âmbito da educação para a saúde.

Os hábitos alimentares foram um dos temas mais desenvolvidos pelos professores estudados (tabela 2), demonstrando como o professor de Educação Física já compreendeu que a discussão nutricional faz parte dos conteúdos a serem discutidos em sua prática pedagógica.

Tabela 2 - Presença dos Temas sobre a Dimensão Alimentação na Aulas dos Docentes, SEMED/MD

\begin{tabular}{lccc}
\hline \multicolumn{1}{c}{ Item } & Escore & Desvio Padrão & Avaliação \\
\hline Consumo de frutas, legumes e verduras & 2,45 & 0,60 & Positiva \\
\hline Consumo de sal, açúcar e gordura & 2,45 & 0,68 & Positiva \\
\hline $\begin{array}{l}\text { Consumo de alimentos ultraprocessados e } \\
\text { fast food }\end{array}$ & 2,41 & 0,85 & Positiva \\
\hline $\begin{array}{l}\text { Importância da ingestão de água } \\
\text { Momento da alimentação e preparo dos } \\
\text { alimentos }\end{array}$ & 1,65 & 0,60 & Positiva \\
\hline
\end{tabular}

Fonte: Rocha; Caetano, 2019.

Conforme destacou Araújo (2010), alimentação inadequada é um dos fatores contribuintes para obesidade dos escolares, contribuindo para o aumento de risco e prevalência de várias doenças crônicas em outras fases da vida dos escolares. Buboiz e colaboradores (2018) avaliaram o consumo alimentar de adolescentes em escolas rurais públicas do Rio Grande do Sul e perceberam que entre 15 a $31 \%$ dos escolares tinham consumido alimentos não saudáveis há pelo menos três dias antes da aplicação do questionário de Vigilância Alimentar e Nutricional.

Em estudo desenvolvido por Batista et al. (2018), na cidade de Itapevi - SP, concluiu-se que 67\% das escolas realizavam atividades curriculares sobre os temas de alimentação e atividade física. Apesar disso, 30,6\% dos alunos têm excesso de peso. Muitos pais possuem conhecimento sobre os males desses 
alimentos à saúde dos seus filhos; entretanto, devido à falta de recursos, acabam por optar pelo lanche de mais fácil acesso para seus filhos.

A discussão sobre preparo e realização da alimentação foi a que apresentou o menor resultado (1,65). Segundo o Guia Alimentar da População Brasileira (2018 BRASIL, 2014), o momento da alimentação tem sido compartilhado com outros afazeres da vida moderna, sobretudo com o tempo de tela, tornando a alimentação uma prática vazia de sentido social. Por outro lado, alimentar-se em grupo e fazer parte do processo de preparo dos alimentos é um fator que influencia positivamente na saúde dos indivíduos.

Tabela 3 - Presença dos Temas sobre a Atividade Física na Aulas dos Docentes, SEMED/MD

\begin{tabular}{lcccc}
\hline Item & Escore & Desvio Padrão & Avaliação \\
\hline $\begin{array}{l}\text { Importância da Atividade Física para a } \\
\text { saúde }\end{array}$ & 2,85 & 0,36 & Positiva \\
\hline $\begin{array}{l}\text { Características e efeitos do } \\
\text { sedentarismo }\end{array}$ & 2,55 & 0,60 & Positiva \\
\hline $\begin{array}{l}\text { Incentivo à prática de Atividade Física } \\
\text { fora da escola }\end{array}$ & 2,65 & 0,67 & Positiva \\
\hline $\begin{array}{l}\text { Impacto da tecnologia na inatividade } \\
\text { física }\end{array}$ & 2,30 & 0,73 & Positiva \\
\hline
\end{tabular}

Fonte: Rocha; Caetano, 2019.

Além da alimentação, outro causa, que contribui fortemente para o crescimento de comportamentos de risco e morbidades em crianças e adolescentes, é o comportamento sedentário. Muitas crianças e jovens não apresentam tempo de prática de atividade física adequados para a idade. O estudo Projeto Nacional de Saúde do Escolar (PeNSE) observou que apenas 43,1\% dos adolescentes eram considerados fisicamente ativos. Diante disso, Guedes e Guedes (1996), afirmaram que a prática da atividade física, através da educação física escolar, pode estimular uma vida saudável na fase adulta.

Dentre os itens analisados na dimensão atividade física, conforme aponta tabela 3, grande parte dos professores estudados abordaram a influência da tecnologia no aumento do comportamento sedentário dos indivíduos $(2,65)$ e assinalaram apontar a importância da atividade física para saúde dos escolares (2,85). Conforme afirmam Lima, Malacarne e Strieder (2012), os professores demonstraram preocupações relativas à saúde de seus alunos, repetindo o discurso sobre a necessidade de terem uma boa refeição, de não permanecerem muito tempo assistindo TV ou jogando videogames, de frequentarem as aulas de educação física e de procurarem outras práticas corporais de movimento em momentos extraclasse. 
Tabela 4 - Presença dos Temas sobre Comportamento de Risco na Aulas dos Docentes, SEMED/MD

\begin{tabular}{lccc}
\hline \multicolumn{1}{c}{ Item } & Escore & Desvio Padrão & Avaliação \\
\hline Consumo e efeitos de drogas lícitas & 1,95 & 1,09 & Regular \\
\hline Doenças sexualmente transmissíveis & 1,35 & 1,26 & Regullar \\
\hline Consumo e efeitos de drogas ilícitas & 2,05 & 1,23 & Positiva \\
\hline Sexo e saúde reprodutiva & 1,45 & 1,19 & Regullar \\
\hline Importância das vacinas para saúde & 1,30 & 1,12 & Regullar \\
\hline $\begin{array}{l}\text { Características e efeitos da } \\
\text { obesidade }\end{array}$ & 2,55 & 0,75 & Positiva \\
\hline $\begin{array}{l}\text { Características e efeitos da } \\
\text { hipertensão arterial }\end{array}$ & 2,10 & 0,96 & Positiva \\
\hline $\begin{array}{l}\text { Características e efeitos da diabetes } \\
\text { Características e efeitos do câncer }\end{array}$ & 2,20 & 0,89 & Positiva \\
\hline $\begin{array}{l}\text { Uso de medicação sem prescrição } \\
\text { médica }\end{array}$ & 1,30 & 1,03 & Regullar \\
\hline Importância da higiene pessoal & 2,55 & 1,03 & Regular \\
\hline
\end{tabular}

Fonte: Rocha; Caetano, 2019.

O sedentarismo é um dos comportamentos de risco mais encontrados na atualidade em crianças e jovens, mas não é o único. Quando se fala nos temas em saúde abordados na escola, cresce o diálogo sobre evitar comportamentos de risco e estimular os hábitos saudáveis. Os resultados encontrados para os comportamentos de risco (tabela 4) mostram que os professores têm desenvolvido poucas atividades sobre doenças sexualmente transmissíveis (1,35); importância das vacinas para saúde $(1,30)$; características e efeitos do câncer (1,30); consumo e efeitos de drogas ilícitas $(1,95)$; doenças sexualmente transmissíveis $(1,45)$; e uso de medicação sem prescrição médica $(1,35)$.

Para Melo e Borges (2017), os hábitos saudáveis evitam doenças relacionadas aos cuidados com o corpo, como o uso de proteção no trabalho e no lazer, utilização de protetor solar, redução de consumo de drogas lícitas e ilícitas, prevenção de infecções sexualmente transmissíveis (IST). Cresce entre os jovens no Brasil as IST's, principalmente o HIV, dados que vem alertando os professores para que esse tema volte a ser abordado dentro da escola.

Outra doença com crescentes números é o câncer, principalmente os cânceres de pele, garganta, pulmão e intestino, provenientes de hábitos prejudiciais à saúde. Um estudo realizado por Giacommozi et. al. (2012) procurou investigar o uso de álcool e outras drogas e as vulnerabilidades relacionadas em adolescente em Florianópolis/ SC, e os resultados mostraram que 30,1\% dos adolescentes usavam álcool; 20,1\%, tabaco; $7 \%$, maconha; $1,3 \%$, cocaína; $0,6 \%$, crack e que estudantes que usavam álcool e drogas faltam mais aulas, participam de mais de brigas e são sexualmente mais ativos. 
Tabela 5 - Presença dos Temas sobre Controle do Stress nas Aulas dos Docentes, SEMED/ MD

\begin{tabular}{lccc}
\hline \multicolumn{1}{c}{ Item } & Escore & Desvio Padrão & Avaliação \\
\hline $\begin{array}{l}\text { Características e efeitos da depressão e da } \\
\text { ansiedade }\end{array}$ & 1,09 & 1,07 & Regular \\
\hline $\begin{array}{l}\text { Importância de estimular o cultivo de amizades e } \\
\text { de relações interpessoais }\end{array}$ & 2,45 & 0,82 & Positiva \\
\hline $\begin{array}{l}\text { Prática de atividades em grupo no } \\
\text { bairro, na escola e em instituições } \\
\text { religiosas }\end{array}$ & 2,35 & 0,81 & Positiva \\
\hline Características e prevenção ao suicídio & 1,65 & 0,93 & Regullar \\
\hline Combate ao bullying na escola. & 2,35 & 0,87 & Positiva \\
\hline Efeitos da privação do sono & 1,30 & 0,97 & Regular \\
\hline
\end{tabular}

Fonte: Rocha; Caetano, 2019.

Os comportamentos de risco podem ser agravados por fatores de estresse crescentes na geração atual. Na dimensão Controle do Estresse (tabela 5), os itens que tiveram menores resultados foram a privação do sono $(1,30)$; depressão e ansiedade $(1,09)$; e prevenção ao suicídio $(1,65)$.

Atualmente, na mídia muitos são os relatos de jovens desenvolvendo quadro de depressão e ansiedade, levando esses jovens a automutilação e suicídio. Segundo Margis (2003), o controle do estresse tornou-se importante porque está relacionado ao bem-estar psíquico, quando não controlado pode contribuir para o desenvolvimento de doenças como o transtorno de pânico, asma, enxaqueca, obesidade e depressão, provocando ações prejudiciais para o indivíduo e para todos que convivem em seu ambiente. Da mesma forma, a privação do sono contribui para que o indivíduo tenha maior probabilidade de desenvolver quadros de depressão e ansiedade, além de obesidade.

O item dessa dimensão com maior escore foi a Importância de estimular o cultivo de relacionamentos interpessoais, visto que fazer e manter bons relacionamentos e boas amizades contribui para a sensação de pertencimento, de estar bem consigo, características fortemente presentes nas aulas de educação física (MELO; BORGES, 2017).

A dimensão "Sociedade e Natureza" (tabela 6) abordou temas transversais para uma promoção da saúde em seus aspectos socioculturais. Entre os itens estavam o controle de finanças, questões sobre criminalidade, violência, preconceito, sustentabilidade e cultura da paz. Os dados apresentaram, pela primeira vez, um escore negativo (importância do controle das finanças) na presença de temas em saúde, no entanto, em termos gerais, a presença é regular das temáticas sobre sociedade e natureza nas aulas de educação física dos docentes analisados. 
Segundo a OMS (2002), a violência é um importante fator interveniente nas condições de saúde dos sujeitos e um problema de saúde pública. A violência faz parte de uma teia de relações, onde o preconceito está no centro dessa discussão. A criminalidade é fruto da violência e as ações contra o incentivo a violência faz parte do processo de promoção da saúde social dos indivíduos, além de que a violência nos bairros afasta os jovens de atividades físicas ao ar livre e de práticas de lazer e socialização com seus pares, causando prejuízos à saúde mental e física (OLIVEIRA, 2015).

Tabela 6 - Presença dos Temas sobre Sociedade e Natureza na Aulas dos Docentes, SEMED/MD

\begin{tabular}{lccc}
\hline Item & Escore & Desvio Padrão & Avaliação \\
\hline Criminalidade e violência no bairro. & 1,80 & 1,00 & Regullar \\
\hline Violência doméstica e de gênero. & 1,30 & 0,97 & Regular \\
\hline $\begin{array}{l}\text { Preconceito racial, de gênero, orientação sexual e/ou } \\
\text { intolerância religiosa. }\end{array}$ & 1,95 & 1,05 & Regullar \\
\hline $\begin{array}{l}\text { Atitudes discriminatórias para com } \\
\text { pessoas com deficiência. }\end{array}$ & 2,25 & 0,85 & Positiva \\
\hline Estimular a cultura da paz. & 2,55 & 0,82 & Positiva \\
\hline Importância do controle de finanças. & 0,90 & 1,02 & Negativa \\
\hline $\begin{array}{l}\text { Responsabilidade das instituições governamentais na } \\
\text { prevenção, tratamento e promoção da saúde. }\end{array}$ & 1,35 & 0,98 & Regullar \\
\hline $\begin{array}{l}\text { Uso dos espaços públicos para prática de atividades } \\
\text { físicas e de lazer. }\end{array}$ & 2,25 & 0,91 & Positiva \\
\hline $\begin{array}{l}\text { Preservação do meio ambiente e } \\
\text { sustentabilidade. }\end{array}$ & 1,85 & 1,08 & Regullar \\
\hline $\begin{array}{l}\text { Exercer a cidadania, ética e } \\
\text { responsabilidade social. }\end{array}$ & 2,30 & 0,80 & Positiva \\
\hline
\end{tabular}

Fonte: Rocha; Caetano, 2019.

A saúde social dos sujeitos também é abordada a partir do estímulo ao controle de finanças, o tema deve estar presente nos currículos das escolas em diversas disciplinas, visto a necessidade em aprender a ter responsabilidade financeira para administrar seus recursos no futuro e não fazerem parte das estatísticas da população brasileira endividada (BRASIL, 2018).

Para mais, a violência não é o único fator que afasta os jovens das práticas de atividade física ao ar livre. Contribuindo para a redução da saúde social dos indivíduos, a poluição e a não preservação do meio ambiente tem destruído diversos ambientes naturais antes utilizados pelas famílias como espaço de atividade física e lazer. Cabe à educação física um papel importante na construção desse conhecimento, visto que a maioria das práticas referentes à cultura corporal de movimento são realizadas em ambiente abertos (BRASIL, 2018). As crianças e os jovens precisam aprender a cultivar hábitos de preservação do meio ambiente e a usufruir dos seus recursos de forma sustentável. 
Na dimensão "Corpo e Saúde", buscou-se compreender o corpo enquanto elemento primordial da educação física nos aspectos físicos, sociais e mentais. O corpo físico e a consciência corporal foram temas frequentemente abordados nas aulas de educação física, conforme a Tabela 7, entretanto o corpo real percebido pelos sujeitos é pouco abordado nas aulas e apresenta características preocupantes na saúde dos escolares.

Tabela 7 - Presença dos Temas sobre Corpo e Saúde na Aulas dos Docentes, SEMED/MD

\begin{tabular}{lccc}
\multicolumn{1}{c}{ Item } & Escore & Desvio Padrão & Avaliação \\
\hline Consciência corporal & 2,55 & 0,68 & Positiva \\
\hline Imagem corporal & 2,25 & 0,91 & Positiva \\
\hline Distúrbios físicos e corporais & 2,15 & 0,98 & Positiva \\
\hline Controle do peso & 2,30 & 0,86 & Positiva \\
\hline Procedimentos de primeiros socorros & 1,80 & 1,19 & Regular \\
\hline
\end{tabular}

Fonte: Rocha; Caetano, 2019.

Os aspectos socioantropológicos sobre o corpo precisam ser amplamente discutidos nas aulas, a fim de criticar conceitos de beleza impostos pela sociedade midiática para um padrão determinado. Além disso, os debates também devem incluir reflexões sobre o culto ao corpo em exagero, associado a patologias psicológicas/ psiquiátricas, que causam distorção da imagem corporal e insatisfação com o corpo reconhecido como a anorexia, bulimia e vigorexia (BUCALEN, 2013).

O item de menor escore na dimensão (tabela 7) refere-se a procedimentos de primeiros socorros $(1,8)$. Os professores devem capacitar os escolares para que conheçam os números emergenciais e essas entidades também devem propiciar aos escolares conhecimentos de primeiros socorros em diferentes tipos de traumas.

Na dimensão Testes e Medidas observaram-se as menores abordagens nas aulas por parte dos professores (tabela 8). Os testes antropométricos fazem parte da estrutura histórica do profissional de educação física; porém, têm se afastado cada dia mais da escola. Os professores relatam a ausência de material, de espaço e a grande quantidade de alunos como justificativas que impossibilitam a realização das avaliações. Contudo, esses testes não dizem respeito somente à avaliação didática sobre os conteúdos abordados nas aulas, mas também servem como indicadores de saúde dos alunos (ARAÚJO et. al, 2010).

Ao utilizar os dados antropométricos, os professores podem detectar a prevalência da enfermidade e orientar os alunos nas aulas sobre a prática regular de atividades físicas, alimentação saudável, hábitos saudáveis e, nos casos mais graves, sensibilizar os pais a buscar tratamentos. Por mais que nem todos os testes possam ser realizados na escola, devido à alta complexidade, atualmente, existem diversos testes de 
baixo custo, e validados cientificamente, capazes de avaliar a aptidão física relacionada à saúde dos alunos, como o PROESP-BR. Alguns atributos da aptidão física relacionada à saúde são aqueles que mostram alguma associação com melhores indicadores de morbimortalidade e/ ou melhor capacidade para desenvolver atividades diárias, por exemplo testes de aptidão cardiorrespiratória, força e resistência muscular, a flexibilidade e a composição corporal (KNUTH, 2014).

Tabela 8 - Presença dos Temas sobre Testes e Medidas na Aulas dos Docentes, SEMED/ MD

\begin{tabular}{lccc}
\multicolumn{1}{c}{ Item } & Escore & Desvio Padrão & Avaliação \\
\hline Teste de flexibilidade & 1,40 & 1,09 & Regullar \\
\hline Teste de resistência abdominal & 1,30 & 1,03 & Regullar \\
\hline Teste de flexão de braço & 1,15 & 0,98 & Regular \\
\hline Peso e estatura & 1,75 & 1,01 & Regullar \\
\hline Teste de escala de silhueta & 0,80 & 0,83 & Negativa \\
\hline Aferição da frequência cardíaca & 1,15 & 1,08 & Regullar \\
\hline Aferição da pressão arterial & 0,80 & 0,95 & Negativa \\
\hline Dobras cutâneas. & 0,40 & 0,59 & Negativa \\
\hline Testes de desvios posturais & 0,90 & 1,07 & Negativa \\
\hline KTK & 0,40 & 0,68 & Negativa \\
\hline PROESP & 0,50 & 0,82 & Negativa \\
\hline
\end{tabular}

Fonte: Rocha; Caetano, 2019.

Em termos de limitação do estudo, pode-se apontar a utilização de um questionário não validado para aquisição das informações, ação que, recentemente, tem sido desenvolvida pela equipe do projeto para melhor consolidação de dados futuros. Além disso, houve a não avaliação aprofundada no aspecto da formação dos docentes, tendo em vista a influência da prática pedagógica a partir dos currículos que os profissionais são egressos. Destaca-se, também, o baixo número de professores entrevistados, podendo não refletir a realidade educacional de todo o município investigado. Ressalta-se, inclusive, a presença de muitos professores com vínculo temporário com a instituição de ensino, implicando em ações pedagógicas perenes. E, por fim, houve uma pouca possibilidade de interlocução com as pesquisas na área, tendo em visto, sobretudo, a investigação sobre a saúde na escola debruçar-se mais nos escolares e não nos docentes, apontando para um campo vasto para o desenvolvimento de investigação.

\section{Conclusão}

Considerando a saúde um tema fundamental a ser abordado nas aulas de educação física e a atuação do professor nas escolas importante, como agente construtor/ transformador de hábitos de vida e 
na formação de cidadãos críticos e conscientes, percebeu-se que os temas em saúde analisados, muitas vezes, não apresentam frequência adequada.

Os temas em saúde denominados Comportamentos de Risco, Controle do Estresse, Sociedade e Natureza e Testes e Medidas apresentaram resultados menos frequentes, revelando dimensões que não apareciam com frequência nos conteúdos trabalhados durante as aulas dos professores de educação física da rede municipal de Marechal Deodoro - AL. Diferente dos temas Alimentação, Atividade Física e Corpo e Saúde, que apresentaram resultados positivos nas práticas pedagógicas dos docentes estudados.

Tais resultados podem apontar para a necessidade de uma rediscussão sobre os conteúdos em educação para saúde nos cursos de graduação nas universidades, bem como um imperativo de ampliação de cursos de formação continuada para docentes no mercado de trabalho, para o desenvolvimento de outras perspectivas de atuação profissional. Além disso, a construção e ampliação de programas de intervenção que estreitam as relações entre universidade e profissionais da Educação Básica poderão auxiliar na melhoria do ensino da educação física e na melhor aproximação do papel docente como promotor da saúde e agente de formação de pessoas fisicamente ativas e conscientes na construção de comportamentos saudáveis nos demais ciclos da vida. Por fim, a urgente necessidade da realização de estudos visando à avaliação das práticas pedagógicas em educação para a saúde, a partir dos docentes e não dos escolares, contribui para a transformação da postura docente em uma ação política, consciente e propositiva para formar pessoas conscientes de maneira pessoal, social e institucional, para melhorar as suas condições de saúde.

\section{Referências}

ARAÚJO, R. A. et al. O papel da Educação Física escolar diante da epidemia da obesidade em crianças e adolescentes. Educação Física em Revista. Vol.4, N² mai/jun/jul/ago- 2010.

BANKOFF, A. D.; ZAMAI, C.A. Estudo do Perfil dos Professores do Ensino Fundamental do Estado de São Paulo sobre o Conhecimento do Tema Atividade Física e Saúde. Revista Brasileira de Atividade Física e Saúde, 4 (30), 1999: 35-42.

BATISTA M.D; MONDINI, L. JAIME P.C. Ações do Programa Saúde na Escola e da alimentação escolar na prevenção do excesso de peso infantil: experiência no município de Itapevi, São Paulo, Brasil, 2014. Epidemiol. Serviço Saúde. 2017; 26(3):569-578.

BRASIL. Lei de Diretrizes e Bases da Educação Nacional, n 9.394/1996. Brasília: Diário Oficial, 20 de dezembro de 1996.

BRASIL. Ministério da Educação. Base Nacional Comum Curricular. Brasília: MEC, 2018. Disponível em: http://basenacionalcomum.mec.gov.br/abase/. Acesso em: 26 de nov. 2019. 
BRASIL. Ministério da Saúde. Diretrizes Nacionais para a Atenção Integral à Saúde de Adolescentes e Jovens na Promoção, Proteção e Recuperação da Saúde. Brasília: Ministério da Saúde, 2010.

BRASIL. Ministério da Saúde. Guia Alimentar para a População Brasileira. Secretária de atenção à saúde. 2 ed. Brasília: 2014.

BRASIL. Ministério da Saúde. Organização Pan-Americana da Saúde. Escola Promotora de Saúde: experiências do Brasil. Brasília: 2007.

BRASIL. Ministério da Saúde. Política Nacional de Atenção Integral à Saúde da Criança: orientações para implementação. Brasília: 2018.

BRASIL, Secretaria de Educação Fundamental. Parâmetros Curriculares Nacionais. Brasília: MEC/SEF, 1998.

BUBOIZ, C.T.R.; ROMBALDI, A.J.; GONZALES, N.G.; AZEVEDO, M.R.; MADRUGA, S.W. Consumo Alimentar conforme o Tipo de Alimentação Consumida em Escolar de Zona Rural do Sul do Brasil. Ciência \& Saúde Coletiva, 23 (8), 2018.

BUCALEN, C. K. et al. Saúde na escola: educação, saúde e inclusão em adolescentes brasileiros. Revista sobre La Infancia y La Adolescencia, 4, 78-90 - Marzo. 2013. ISSN 2174- 7210.

COLETIVO DE AUTORES. Metodologia do Ensino de Educação Física. São Paulo: Cortez, 1992.

DALMORO, M. \&; VIEIRA, K.M. Dilemas na Construção de Escalas Tipo Likert: O Número de Itens e a Disposição Influenciam os Resultados? Revista Gestão Organizacional, 6 - Edição Especial, 2013.

GUEDES, D. P.; GUEDES, J. E. R. P. Subsídios para implementação de programas direcionados a promoção da saúde através da Educação Física Escolar. Revista da Associação de Professores de Educação Física de Londrina. 8(15), 3-11. 1996.

GUIMARÃES, C.C.P.A.; NEIRA, M.G; VELARDI, M. Reflexões sobre Saúde e Educação Física Escolar: a Visão dos Professores. Revista Hipótese, 1(4), 2015: 113-138.

GIACOMOZZI, A. I. et al. Levantamento sobre uso de álcool e outras drogas e vulnerabilidades relacionadas de estudantes de escolas públicas participantes do programa saúde do escolar/saúde e prevenção nas escolas no município de Florianópolis. Saúde Sociedade. 2012. 21(3):612-622.

IBGE. Pesquisa Nacional de Saúde do Escolar. Rio de Janeiro: IBGE, 2015.

JESUS, R.F.; SAWITZKI, R.L. Entendimentos de Professoras Unidocentes sobre Tema Saúde: Apontamentos frente à Formação Inicial/Continuada, Planos de Estudos e Planejamentos Docentes em uma Escola Estadual do RS. Conexões, 13(4), 2015: 49-78.

KNUTH et al. "Saúde é o que interessa, o resto não tem pressa"? Um ensaio sobre educação física e saúde na escola. Revista Brasileira Atividade Física e Saúde • Pelotas/RS • 19(4):429-432 • Jul./2014. 
KNUTH, A.G.; AZEVEDO, M.R.; RIGO, L.C. A Inserção de Temas Transversais em Saúde nas Aulas de Educação Física. Revista Brasileira de Atividade Física e Saúde, 12(3) 2006: 73-78.

LIMA, D. F.; MALACARNE, V.; STRIEDER, D. M. O papel da escola na promoção da saúde - uma mediação necessária. EccoS - Revista Cientifíca. São Paulo, n. 28, p. 191-206, maio/ago. 2012.

LUCIAN, R. Repensando o uso da escala Likert: tradição ou escolha técnica. PMKT - Revista Brasileira de Pesquisas de Marketing, Opinião e Mídia (PMKT on-line) | ISSN 2317-0123 | São Paulo, v. 9, n. 1, p. 12-28, jan.-abr. 2016.

MARGIS, R. et al. Relação entre estressores, estresse e ansiedade. Revista de Psiquiatria do Rio Grande do Sul, 2003. 25(1), 65-74.

MELO, I.G.; BORGES, C.J. As Contribuições Da Educação Física Escolar Na Formação Do Estilo De Vida Dos Alunos. Atividade física e promoção da saúde na escola: coletânea de estudos. Fortaleza: EdUECE, 2017. ISBN: 978-85-7826-540-3

MINAYO, M. C. S. Ciência, técnica e arte: o desafio da pesquisa social. Pesquisa social: teoria, método e criatividade. Petrópolis: Vozes, 1994.

OLIVEIRA et al. Projetos e Práticas Em Educação Para a Saúde na Educação Física Escolar: Possibilidades! Revista Educação Física/UEM, v. 26, n. 2, p. 243-255, 2. trim. 2015.

OLIVEIRA, V. J. M. e et al. Educação para a saúde na educação física escolar: uma questão pedagógica. Cadernos de Formação RBCE, p. 68-79, set. 2014.

ORGANIZAÇÃO MUNDIAL DA SAÚDE. Constituição da Organização Mundial da Saúde. Documentos básicos, suplemento da $45^{\mathrm{a}}$ edição, outubro de 2006. Disponível em espanhol em: http://www.who.int/governance/eb/who_constitution_sp.pdf. Acesso em 04 de nov. 2019.

Organização Mundial da Saúde. Relatório Mundial sobre violência e saúde. Geneve. 2002.

PAIXÃO, J.A.; AGUIAR, C.M.; SILVEIRA, F.S.A. Percepção da Obesidade Juvenil entre Professores de Educação Física na Educação Básica. Revista Brasileira Medicina do Esporte, 22 (6), 2016: 501-505.

REIS, E.A.; REIS I.A. Análise Descritiva de Dados. Relatório Técnico do Departamento de Estatística da UFMG. 2002. Disponível em: www.est.ufmg.br. Acesso em 07 de nov. de 2019.

ROMBALDI, A.J.; BORGES, T.T.; CANABARRO, L.K.; CORRÊA, L.Q.; NEUTZLING, M.B. Conhecimento de Professores de Educação Física sobre Fatores de Risco para Doenças Crônicas de uma Cidade do Sul do Brasil. Revista Brasileira de Cineantropometria e Desempenho Humano, 14(1), 2012: 61-72.

TEIXEIRA, B.A.; COLOMBO, B.D. A Temática da Saúde na Educação Física no Ensino Médio: um Estudo com Professores Estaduais de Sombrio/SC. Revista Kinesis, 38, 2020: 01-13.

ZANCHA, D.; MAGALHÃES, G.B.S; MARTINS, J.; SILVA, T.A.; ABRAHÃO, T.B. Conhecimento dos Professores de Educação Física Escolar sobre a Abordagem Renovada e a Temática Saúde. Conexões, 11 (1), 2013: 204-217. 


\section{Como citar este artigo}

ROCHA, M. M.; CAETANO, A. F. P. Temáticas em Saúde na Prática Pedagógica de Professores de Educação Física de um Município do Estado de Alagoas (Brasil). Revista Kinesis, Santa Maria, v. 39, p.01-18, 2021.

* O presente trabalho não contou com apoio financeiro de nenhuma natureza para sua realização. 